\title{
Mercury vapour exposure: a study of dental students
}

\author{
Blood mercury levels of dental students and dentists at a dental school by H. Tezel, O. S. Ertas, F. Ozata, C. Erakin, and \\ A. Kayali Br Dent J 2001; 191: 449-452
}

\section{Objective}

To determine the blood mercury levels in dental students and clinical teaching staff in a dental school using amalgam as a restorative material.

\section{Setting \\ A dental school in Ege University, Turkey surveyed during one academic year.}

\section{Subjects and methods \\ Cross-sectional study of groups of dental students $(n=92)$ in years I to $\mathrm{V}$, clinical teachers in restorative dentistry $(\mathrm{n}=16)$ and controls $(n=14)$. Mercury concentration was estimated in venous blood samples using a cold vapour atomic absorption method at the commencement and end of the academic year. Daily air mercury levels were determined in clinical and teaching areas by measuring the darkening of palladium chloride discs using spectrophotometry.}

\section{Results}

here were statistically significant increases $(\mathrm{p}<0.001)$ in plasma mercury concentration between measurements in all groups at the end of the academic year. Red cell mercury levels were also consistently elevated. Although the highest levels of mercury were recorded in persons working with amalgam, increased levels were also found in subjects working in the teaching classrooms but not with amalgam (controls and first year students).

\section{Conclusion}

Increased mercury levels appeared to be due to background exposure from spillage of mercury and amalgam residues on floors. Increased mercury hygiene and regular control of working atmosphere should be implemented to prevent mercury exposure in the dental pre-clinical laboratory.

\section{Comment}

The potential dangers to dental person1 nel arising from the use of amalgam are well documented yet there is a curious lack of information regarding the hazards that may arise in dental schools and hospitals. This paper therefore should give food for thought to those responsible for the training of the dentists of the future.

The paper reports the levels of mercury found in plasma and whole blood of dental students and their clinical teachers at the University of Ege at the beginning and end of the academic year. Also there was included in the study a control group comprising fulltime clinical teachers who worked in the periodontal department and who therefore had no direct contact with amalgam. Individuals who smoked, ate fish or consumed alcohol were excluded from the study. Furthermore none of the participants had amalgam fillings placed during the study period.

Levels of mercury in blood were assessed using atomic absorption spectrophotometry. Samples were centrifuged to allow for the determination of levels in plasma and packed red cells. The results were extrapolated to give values for whole blood
Second year students had the highest levels of mercury in plasma at the beginning of the study $(17.78 \mu / \mathrm{l})$. Curiously the 'fresh' first year students were found to have the second highest baseline values for mercury in plasma $(13.99 \mu / 1)$ in spite of their having had no previous experience in handling amalgam. The remaining students, the teachers and the control group all showed similar baseline values with respect to plasma $(10.20 \mu / 1-11.24 \mu / 1)$. However in the case of the teachers the levels in whole blood was greater than in the other groups $(15.69 \mu / \mathrm{l})$. Values for plasma reflect current exposure whilst values for red cells (and therefore whole blood) are determined in part by body burden. Thus the baseline for the teachers is partly a consequence of their exposure to mercury over their practising career.

As might be expected the levels of mercury in the blood of each year of students increased throughout the academic year although they did not reach values which indicate toxicity. In this school, first year students work in a teaching laboratory shared with their more senior colleagues undertaking phantom head courses. They too exhibited increased levels of mercury in spite of their not being directly involved in the use of amalgam. The increase was not as great as those working with amalgam but was similar to that of the control group.

The elevation of mercury levels in the control group in spite of their not working with amalgam, was probably a result of percolation of vapour from other sites in the building.

The authors had also assessed the average mercury vapour levels in the teaching clinics including the periodontal department and the multi-purpose teaching laboratory. All showed levels of vapour that, with the exception of the periodontal department, were in excess of the current WHO threshold limit value of $24 \mu \mathrm{gHg} / \mathrm{m}^{3}$ air.

In the light of these findings the authors pointed to the need for improved mercury hygiene within the school.

\section{Eoin R. Smart}

Lecturer in Conservative Dentistry, The Dental School, University of Newcastle upon Tyne 